\title{
The Exact Hausdorff Measure of the Zero Set of Fractional Brownian Motion
}

\author{
D. Baraka - T.S. Mountford
}

Received: 30 April 2009 / Revised: 12 October 2009 / Published online: 6 January 2010

(C) Springer Science+Business Media, LLC 2010

Abstract Let $\left\{X(t), t \in \mathbb{R}^{N}\right\}$ be a fractional Brownian motion in $\mathbb{R}^{d}$ of index $H$. If $L(0, I)$ is the local time of $X$ at 0 on the interval $I \subset \mathbb{R}^{N}$, then there exists a positive finite constant $c(=c(N, d, H))$ such that

$$
m_{\phi}\left(X^{-1}(0) \cap I\right)=c L(0, I)
$$

where $\phi(t)=t^{N-d H}\left(\log \log \frac{1}{t}\right)^{d H / N}$, and $m_{\phi}(E)$ is the Hausdorff $\phi$-measure of $E$. This refines a previous result of Xiao (Probab. Theory Relat. Fields 109: 126-197, 1997 ) on the relationship between the local time and the Hausdorff measure of zero set for $d$-dimensional fractional Brownian motion on $\mathbb{R}^{N}$.

Keywords Local times · Hausdorff measures · Level sets · Fractional Brownian motion

Mathematics Subject Classification (2000) 60G60 • 60G15 · 60G17

\section{Introduction}

Let $\left\{Y(t) ; t \in \mathbb{R}^{N}\right\}$ be a fractional Brownian motion in $\mathbb{R}$ of index $H(0<H<1)$, i.e., the centered, real-valued Gaussian random field with covariance function

$$
E(Y(t) Y(s))=\frac{1}{2}\left(|t|^{2 H}+|s|^{2 H}-|t-s|^{2 H}\right),
$$

where $|\cdot|$ is the Euclidean norm in $\mathbb{R}^{N}$.

D. Baraka · T.S. Mountford $(\bowtie)$

Département de Mathématiques, École Polytechnique Fédérale, 1015 Lausanne, Switzerland

e-mail: thomas.mountford@epfl.ch 
It is well known (see, e.g., [14]) that $Y$ is a self-similar process of exponent $H$, i.e., for any $a>0$,

$$
Y(a \cdot)={ }^{d} a^{H} Y(\cdot),
$$

and has stationary increments, that is, for every $b \in \mathbb{R}^{N}$,

$$
Y(\cdot+b)-Y(b)={ }^{d} Y(\cdot),
$$

where $Z={ }^{d} Y$ means that two processes $Z$ and $Y$ have the same distribution.

In this article we exploit the strong local nondeterminism (strong LND) property of fractional Brownian motion. Explicitly, in [22], Lemma 7.1, it is proved that there exists a constant $c_{1} \in(0, \infty)$, depending on $H$ only, such that for any $t \in \mathbb{R}^{N}$ and $0 \leq r \leq|t|$

$$
\operatorname{Var}(Y(t)|Y(s): r \leq| s-t \mid) \geq c_{1} r^{2 H} .
$$

The concept of local nondeterminism of Gaussian process was introduced in [7]. General conditions for strong LND of Gaussian processes are given in $[5,6,8,9]$, and [22]. We also refer to [10, 19], and [30] for more information on strong LND and its use in studying sample path properties of Gaussian random fields.

We can construct from $\left\{Y(t), t \in \mathbb{R}^{N}\right\}$ a Gaussian random field $\left\{X(t) ; t \in \mathbb{R}^{N}\right\}$ in $\mathbb{R}^{d}$ by

$$
X(t)=\left(X_{1}(t), \ldots, X_{d}(t)\right),
$$

where $X_{1}, \ldots, X_{d}$ are independent copies of $Y$. If $Y$ is a fractional Brownian motion in $\mathbb{R}$ of index $H$, then $\left(X(t) ; t \in \mathbb{R}^{N}\right)$ is called a $d$-dimensional fractional Brownian motion of index $H$. When $N=1$ and $H=1 / 2, X(\cdot)$ is the ordinary $d$-dimensional Brownian motion.

The local time, when it exists, is defined by ( $\mathcal{I}$ is the usual indicator function)

$$
L(x, I)=\lim _{\epsilon \rightarrow 0} \frac{1}{c_{d} \epsilon^{d}} \int_{I} \mathcal{I}_{|X(t)-x| \leq \epsilon} d t
$$

for $x \in \mathbb{R}^{d}$ and $I \subset \mathbb{R}^{N}$ and $c_{d}$ the volume of the unit ball in $d$-dimensional Euclidean space. It is well known (see references immediately below) that there exists a local time for all $x$ and bounded $I \subset \mathbb{R}^{N}$ if and only if $N>d H$. From now on we will suppose that this inequality holds. It follows from [16] (see also [7] and [22]), using the local nondeterminism property, that this local time has a jointly continuous version $L(x, I)$ satisfying certain Hölder conditions in the time and space variables, respectively, and

$$
\int_{I} \xi(Y(s)) d s=\int_{\mathbb{R}} \xi(x) L(x, I) d x
$$

for every bounded measurable function $\xi: \mathbb{R}^{d} \rightarrow \mathbb{R} . L(x, \cdot)$ can be extended to be finite measure supported on the level set

$$
Y_{T}^{-1}(x)=\{t \in T: Y(t)=x\}
$$


see [2]. This fact has been used by [1, 6, 13, 19, 25, 29], and [31] to study the Hausdorff dimension of the level sets, inverse image, and multiple points of stochastic processes.

The aim of this paper is to study the zero set $\{\underline{t}: X(\underline{t})=0\}$ or, more generally, $\{\underline{t}: X(\underline{t})=x\}$ for $x \in \mathbb{R}^{d}$. The exact Hausdorff measure of $X^{-1}(x)$ has been studied in [31] and [33] for the class of strongly locally nondeterministic Gaussian random fields with stationary increments.

Recall that by an exact Hausdorff measure function for a set $E$ is meant a function $\Psi(r)$ defined for small $r \geq 0$, vanishing at the origin, increasing, and continuous and such that the Hausdorff $\Psi$-measure of the set $E$ defined by

$$
m_{\Psi}(E)=\lim _{\delta \rightarrow 0} \inf _{\left\{I_{i}\right\}}\left\{\sum_{i=1}^{\infty} \Psi\left(\left|I_{i}\right|\right): E \subset \bigcup_{i=1}^{\infty} I_{i},\left|I_{i}\right|<\delta\right\}
$$

is almost surely positive and finite, where $|I|$ is the diameter of a set $I$; see [15] or [24].

Essentially, there is at most one correct function $\Psi$ for a given set $E$, in the sense that if $m_{\Psi_{1}}(E) \in(0, \infty)$ and if

$$
\lim _{r \downarrow 0} \frac{\Psi_{1}(r)}{\Psi_{2}(r)}=0, \quad \text { then } \quad m_{\Psi_{2}}(E)=\infty,
$$

while if

$$
\lim _{r \downarrow 0} \frac{\Psi_{1}(r)}{\Psi_{2}(r)}=\infty, \quad \text { then } \quad m_{\Psi_{2}}(E)=0 .
$$

[31] shows (in this more general framework) the following:

Theorem 1.1 Let $\left\{X(t), t \in \mathbb{R}^{N}\right\}$ be a d-dimensional fractional Brownian motion of index $H$. There exists a finite constant $K>0$ such that, for every fixed $x \in \mathbb{R}^{d}$ and all compact intervals $I \subset \mathbb{R}^{N}$, with probability 1 ,

$$
K L(x, I) \leq m_{\phi}(\{s: X(s)=x, s \in I\})<\infty,
$$

where $\phi(h)=h^{N-d H}(\log \log 1 / h)^{d H / N}$.

This identifies the correct Hausdorff measure function.

We will show the following:

Theorem 1.2 Let $\left\{X(t), t \in \mathbb{R}^{N}\right\}$ be a d-dimensional fractional Brownian motion of index $H$. There exists a finite constant $c>0$ (depending only on $H, N$, and $d$ ) such that, with probability 1 ,

$$
m_{\phi}\left(X^{-1}(0) \cap I\right)=c L(0, I),
$$

where $\phi(t)=t^{N-d H}\left(\log \log \frac{1}{t}\right)^{d H / N}$. 
The first result of this kind was due to [28], who showed that if $X$ is a onedimensional Brownian motion $\left(H=\frac{1}{2}, d=N=1\right)$, there exists a positive finite constant $c_{2}$ such that

$$
m_{\phi}(\{s: X(s)=0, s \leq t\})=c_{2} L(0,[0, t]) \quad \text { a.s. },
$$

where $\phi(r)=(r \log \log 1 / r)^{1 / 2}$ for all $t>0$.

Due to [21], much more is known, where the constant $c_{2}$ is identified as $1 / \sqrt{2}$ and, in fact, it is shown that a.s., simultaneously over all $x$,

$$
m_{\phi}(\{s: X(s)=x, s \leq t\})=L(x, t) / \sqrt{2} .
$$

In showing Theorem 1.2 we will extensively use the ideas found in these two pioneering papers.

The Hausdorff dimension of the level sets of fractional Brownian motion and more general Gaussian random fields have been studied by several authors, see [2, 17], and the references therein. The uniform Hausdorff dimension of the level sets and inverse image of strongly locally nondeterministic Gaussian random fields were obtained in [22]. The exact Hausdorff measure of the level sets of certain stationary Gaussian processes was considered in [11] and [12], where partially the method of [28] is adapted, and it is essential to assume stationarity and that $N=1$.

In the paper, for notational and conceptual reasons, we consider the case $N=$ 1 and $d$-dimensional fractional Brownian motion. In the last section we sketch the modifications necessary to deal with general temporal dimension.

The rest of the paper is organized as follows. Section 2 contains the asymptotic relations needed in the sequel. In order to solve some dependence problems that are a major obstacle, we introduce in Sect. 3 independent fractional Brownian motions so that on certain intervals, the local time at zero is close to that of original process. In Sect. 4, we use the approach of [28] by constructing an economic covering and prove the upper bound. In Sect. 5, we prove the lower bound of $m_{\phi}\left(X^{-1}(x) \cap[0,1]\right)$ using Lemma 4 in [28].

We will use $K_{i, j}$ to denote a constant introduced in Section $i$ which will be used later, while $K, K^{\prime}, K^{\prime \prime}, K_{1}, \ldots$ will denote unspecified positive finite constants which may not necessarily be the same in each occurrence.

\section{Preliminary Facts}

Let $\{X(t), t \in \mathbb{R}\}$ be a $d$-dimensional fractional Brownian motion of index $H$. We begin this section with two results from Talagrand and Xiao.

We will need the following lemma from [27].

Lemma 2.1 There exists a finite constant $K_{2,1}<\infty$ such that, for any $n \geq K_{2,1}$, we have

$$
P\left\{\sup _{|s| \leq 2^{-n}}|X(s)| \geq n 2^{-n H}\right\} \leq \exp \left(-n^{2} / K_{2,1}\right) .
$$


In the rest of the paper, we write $L(x, t)$ for $L(x,[0, t])$, the local time of $X$ at $x$ on the interval $[0, t]$. The following lemma is a consequence of [31], Lemma 2.5.

Lemma 2.2 ([31]) There exists a positive and finite constant $K_{2,2}$ such that, for all integers $M \geq 1$, small $r>0, x, y \in \mathbb{R}^{d}$, and $0<\gamma<\min \{1,(1-H d) / 2 H\}$, we have

$$
E\left[|L(x+y, r)-L(x, r)|^{M}\right] \leq K^{M}|y|^{M \gamma} r^{M(1-H(d+\gamma))}(M !)^{H d+2 \gamma} .
$$

This yields, after judicious choice of $M$, the following:

Corollary 2.3 For a fractional Brownian motion $X$ on $\mathbb{R}^{d}$, there exists a constant $g$ strictly greater than zero such that, for small $y \in \mathbb{R}^{d}$ and all $x \in \mathbb{R}^{d}$,

$$
P\left\{|L(x, 1)-L(x+y, 1)| \geq y^{g}\right\} \leq e^{-y^{g}} .
$$

Now we can prove the following:

Proposition 2.4 There exists a finite constant $K_{2,3}<\infty$ such that, for all $n$ large enough and $\frac{n}{4} \leq r \leq \frac{n}{2}$,

$$
P\left\{\sup _{s \in\left[0,2^{-n}[\right.}\left|L\left(X(s), 2^{-r}\right)-L\left(0,2^{-r}\right)\right| \geq 2^{-r(1-d H)}\right\} \leq \exp \left(-\frac{n^{2}}{K_{2,3}}\right) .
$$

Proof Let $n$ be an integer large enough and fix $\frac{n}{4} \leq r \leq \frac{n}{2}$. By Lemma 2.1, showing the desired probability bound in (2.3) reduces to majorizing

$$
P\left\{\sup _{|y| \leq n 2^{-n H}}\left|L\left(y, 2^{-r}\right)-L\left(0,2^{-r}\right)\right| \geq 2^{-r(1-d H)}\right\} .
$$

For all $j \in \mathbb{N}$, let $y_{i, j}^{n}=\frac{i}{2^{j}} n 2^{-n H} \in\left[-n 2^{-n}, n 2^{-n}\right]^{d}, i \in\left\{-2^{j}, \ldots, 2^{j}\right\}^{d} \equiv$ $I(d, j)$.

Using a chaining argument, we have that, for large $n$,

$$
\begin{aligned}
& \left\{\sup _{|y| \leq n 2^{-n H}}\left|L\left(y, 2^{-r}\right)-L\left(0,2^{-r}\right)\right|>2^{-r(1-d H)}\right\} \\
& \subset \bigcup_{j=0}^{\infty} \bigcup_{v=1}^{d} \bigcup_{i \in I(d, j)}\left\{\left|L\left(y_{i, j}^{n}, 2^{-r}\right)-L\left(y_{i-e_{v}, j}^{n}, 2^{-r}\right)\right|>2^{-r(1-d H)} 2^{-j g} 2^{-g n / 3}\right\},
\end{aligned}
$$

where $e_{v}$ is the unit vector in the $v$ th coordinate direction.

With the last expression, we can bound from above, for $n$ large,

$$
A_{n} \equiv P\left\{\sup _{|y| \leq n 2^{-n H}}\left|L\left(y, 2^{-r}\right)-L\left(0,2^{-r}\right)\right| \geq 2^{-r(1-d H)}\right\}
$$


by

$$
\sum_{j=0}^{\infty} \sum_{v=1}^{d} \sum_{i \in I(d, j)} P\left\{\left|L\left(y_{i, j}^{n}, 2^{-r}\right)-L\left(y_{i-e_{v}, j}^{n}, 2^{-r}\right)\right| \geq 2^{-r(1-H)} 2^{-j g} 2^{-g n / 3}\right\} .
$$

By scaling, $P\left\{\left|L\left(y_{i, j}^{n}, 2^{-r}\right)-L\left(y_{i-e_{v}, j}^{n}, 2^{-r}\right)\right| \geq 2^{-r(1-d H)} 2^{-j g} 2^{-g n / 3}\right\}=$ $P\left\{\left|L\left(2^{r H} y_{i, j}^{n}, 1\right)-L\left(2^{r H} y_{i-e_{v}, j}^{n}, 1\right)\right| \geq 2^{-j g} 2^{-g n / 3}\right\}$.

Now $2^{r H}\left|y_{i-e_{v}, j}^{n}-y_{i, j}^{n}\right|=2^{-j} 2^{-H n} n 2^{H r}<2^{-j} 2^{-H n / 3}$ for all $j$ if $n$ is sufficiently large by our restriction of $r$, and so Corollary 2.3 gives

$$
P\left\{\left|L\left(y_{i, j}^{n}, 2^{-r}\right)-L\left(y_{i-e_{v}, j}^{n}, 2^{-r}\right)\right| \geq 2^{-r(1-d H)} 2^{-j g} 2^{-g H n / 3}\right\} \leq e^{-2^{j g} 2^{g H n / 3}}
$$

for all $j>0$ if $n$ is sufficiently large.

In consequence,

$$
\begin{aligned}
& P\left\{\sup _{|y| \leq n 2^{-n H}}\left|L\left(y, 2^{-r}\right)-L\left(0,2^{-r}\right)\right| \geq 2^{-r(1-d H)}\right\} \leq \sum_{j=0}^{\infty} \sum_{v=1}^{d} \sum_{i \in I(d, j)} e^{-2^{j g} 2^{g H n / 3}} \\
& \quad \leq d \sum_{j=0}\left(2^{j+1}+1\right)^{d} e^{-2^{j g} 2^{g H n / 3}} \leq e^{-2^{g H n / 3} / 2},
\end{aligned}
$$

again provided that $n$ is sufficiently large. This bound is sufficient to establish the proposition.

Proposition 2.5 There exists $c_{0}$ a finite constant such that for all $\epsilon>0$, there exists $\delta>0$ and an increasing sequence $V=\left\{n_{i}, i>1\right\}$ of positive integers such that

$$
P\left\{\left(\log n_{i}\right)^{d H} \leq\left(c_{0}+\epsilon\right)^{d H} L(0,1)\right\} \geq \frac{1}{n_{i}^{1-\delta}} \quad \text { for all } n_{i} \in N .
$$

To prove this proposition, we need the result of [32], Theorem 3.23, which is an extension of the main result of [18],

Lemma 2.6 [32] There exist $K_{2,3}$ and $K_{2,4}$ in $(0, \infty)$ such that, for $x$ large enough,

$$
K_{2,3} \leq-\frac{\log P\{L(0,1)>x\}}{x^{N / d H}} \leq K_{2,4}
$$

Proof of Proposition 2.5 Let

$$
c_{0}=\liminf _{x \rightarrow \infty}-\frac{\log P\{L(0,1)>x\}}{x^{1 / d H}} \in(0, \infty) \quad \text { by Lemma } 2.6 ;
$$

then for all $\epsilon>0$, there exists a sequence $x_{i} \nearrow \infty$ such that

$$
-\frac{\log P\left\{L(0,1)>x_{i}\right\}}{x_{i}^{1 / d H}} \leq c_{0}+\epsilon / 2
$$


We can write the last as

$$
P\left\{L(0,1)>x_{i}\right\} \geq e^{-\left(c_{0}+\epsilon / 2\right) x_{i}^{1 / d H}} .
$$

We choose $n_{i}=\exp \left(x_{i}^{1 / d H}\left(c_{0}+\epsilon\right)\right)$. Without loss of generality, we have an increasing sequence $n_{i}$ of positive integers such that

$$
\begin{aligned}
& \text { for all } \epsilon>0, \text { there exist } n_{i} \nearrow \infty \text { such that } \\
& \qquad P\left\{L(0,1) \geq\left(\frac{\log n_{i}}{c_{0}+\epsilon}\right)^{d H}\right\} \geq n_{i}^{-\frac{c_{0}+\epsilon / 2}{c_{0}+\epsilon}} .
\end{aligned}
$$

Let $1-\delta=\frac{c_{0}+\epsilon / 2}{c_{0}+\epsilon}$ and $V=\left\{n_{i}, i>1\right\}$.

Remark [20] show that, in fact, Lemma 2.6 can be extended: the (nontrivial) limit, as $x$ becomes large, of $-\frac{\log P\{L(0,1)>x\}}{x^{N / d H}}$ exists. We have chosen not to exploit this new result since, in fact, the argument given for proving Theorem 1.2 does not need the existence of this limit. We feel that this may be important in cases where this limit is not known to exist.

Remark In fact, [31] treats explicitly the case $N=1$, but the ideas can deal with all temporal dimensions and are written out in this case in [3].

\section{Independent Process}

We use the representation for $X$ a $d$-dimensional fractional Brownian motion of parameter $H$,

$$
X_{i}(t)=c_{H} \int_{\mathbb{R}}\left(\frac{1}{|x-t|^{\alpha}}-\frac{1}{|x|^{\alpha}}\right) d W_{i}(x), \quad i=1, \ldots, d,
$$

where $2 \alpha=1-2 H$, and $W_{i}(\cdot)$ are independent white noises (see, e.g., [26]). If $\alpha=0$, this formula is not true, but in this case $X$ is a Brownian motion and more is known, see, for example, [21] and [23].

First, we will consider the case $d=1$.

In order to solve some dependence problems, we introduce associated processes $\{X(a, b, t) ; t \in \mathbb{R}\}$, see [27] and [4]. Given $0<a<b<\infty$, we define the associated process by

$$
X(a, b, t)=c_{H} \int\left(\frac{1}{|x-t|^{\alpha}}-\frac{1}{|x|^{\alpha}}\right) d Z_{a, b}(x)
$$

and

$$
\begin{aligned}
\dot{Z}_{a, b}(x) & =\dot{W}(x) \quad \text { for } a \leq|x| \leq b \\
& =\dot{W}_{a, b}(x) \quad \text { elsewhere }
\end{aligned}
$$


where $W_{a, b}$ and $W_{a^{\prime}, b^{\prime}}, 0<a<b<a^{\prime}<b^{\prime}$, are independent white noises, also independent of $W$. Most importantly, for $a<b \ll a^{\prime}<b^{\prime}$, the processes $X(a, b, t)$ and $X\left(a^{\prime}, b^{\prime}, t\right)$ are independent.

Let

$$
\begin{aligned}
& \hat{X}(a, b, t)=c_{H} \int_{\{a \leq|x| \leq b\}^{c}}\left(\frac{1}{|x-t|^{\alpha}}-\frac{1}{|x|^{\alpha}}\right) d W(x), \\
& \tilde{X}(a, b, t)=c_{H} \int_{\{a \leq|x| \leq b\}^{c}}\left(\frac{1}{|x-t|^{\alpha}}-\frac{1}{|x|^{\alpha}}\right) d Z_{a, b}(x) .
\end{aligned}
$$

So

$$
\begin{aligned}
& X(t)=c_{H} \int_{\{a \leq|x| \leq b\}}\left(\frac{1}{|x-t|^{\alpha}}-\frac{1}{|x|^{\alpha}}\right) d W(x)+\hat{X}(a, b, t), \\
& X(a, b, t)=c_{H} \int_{\{a \leq|x| \leq b\}}\left(\frac{1}{|x-t|^{\alpha}}-\frac{1}{|x|^{\alpha}}\right) d W(x)+\tilde{X}(a, b, t) .
\end{aligned}
$$

For $n$ large enough and $n / 4 \leq r \leq n / 2$, one is interested in $s, t \in I=\left[0,2^{-r}\right.$, $a=\frac{2^{-r}}{n^{\beta}}$, and $b=2^{-r} n^{\beta}$ for a $\beta>0$ to be fixed later.

Lemma 3.1 For all $s, t \in I$, there exists a positive constant $K_{3,1}$ such that, for $i \in$ $\{1,2, \ldots, d\}$,

$$
E\left[\hat{X}_{i}(a, b, s) \hat{X}_{i}(a, b, t)\right] \leq K_{3,1} \frac{2^{-2 r d H}}{n^{\beta \gamma}}
$$

with $\gamma=2 \min ((1-H), H)$.

Proof

$$
\begin{aligned}
E[\hat{X}(a, b, s) \hat{X}(a, b, t)] \leq & 2 c_{H} \int_{b}^{\infty}\left(\frac{1}{|x-s|^{\alpha}}-\frac{1}{|x|^{\alpha}}\right)\left(\frac{1}{|x-t|^{\alpha}}-\frac{1}{|x|^{\alpha}}\right) d x \\
& +c_{H} \int_{0}^{a}\left(\frac{1}{|x-s|^{\alpha}}-\frac{1}{|x|^{\alpha}}\right)\left(\frac{1}{|x-t|^{\alpha}}-\frac{1}{|x|^{\alpha}}\right) d x \\
& +c_{H} \int_{-a}^{0}\left(\frac{1}{|x-s|^{\alpha}}-\frac{1}{|x|^{\alpha}}\right)\left(\frac{1}{|x-t|^{\alpha}}-\frac{1}{|x|^{\alpha}}\right) d x
\end{aligned}
$$

The first integral in the right part is less than $\frac{2^{-2 r H}}{n^{2 \beta(1-H)}}$. The bounding of the second integral (and in the same way, the third), using the Cauchy-Schwarz inequality, is reduced to bounding the integral

$$
I_{s}=\int_{0}^{a}\left(\frac{1}{|x-s|^{\alpha}}-\frac{1}{|x|^{\alpha}}\right)^{2} d x \leq 2 \int_{0}^{a}\left(\frac{1}{|x-s|^{2 \alpha}}+\frac{1}{|x|^{2 \alpha}}\right) d x
$$


Case 1. $s>2 \cdot \frac{2^{-r}}{n^{\beta}}$ :

$$
I_{S} \leq 4 \int_{0}^{\frac{2^{-r}}{n^{\beta}}} \frac{1}{|x|^{2 \alpha}} d x \leq K_{1} \frac{2^{-2 r H}}{n^{2 \beta H}} .
$$

Case 2. Intermediate:

$$
I_{s} \leq 2 \int_{0}^{\frac{2^{-r}}{n^{\beta}}}\left(\frac{1}{(s-x)^{2 \alpha}}+\frac{1}{x^{2 \alpha}}\right) d x \leq K_{2} \frac{2^{-2 r H}}{n^{2 \beta H}} .
$$

Case 3. $s<\frac{2^{-r}}{n^{\beta}}$ :

$$
\begin{aligned}
I_{s} & \leq 2\left[\int_{0}^{s} \frac{1}{(s-x)^{2 \alpha}} d x+\int_{s}^{\frac{2^{-r}}{n^{\beta}}} \frac{1}{(x-s)^{2 \alpha}} d x+\int_{0}^{\frac{2^{-r}}{n^{\beta}}} \frac{1}{x^{2 \alpha}} d x\right] \\
& \leq \frac{2}{1-2 \alpha}\left[s^{1-2 \alpha}+\left(\frac{2^{-r}}{n^{\beta}}-s\right)^{1-2 \alpha}+\left(\frac{2^{-r}}{n^{\beta}}\right)^{1-2 \alpha}\right] \\
& \leq K_{3}\left(\frac{2^{-r}}{n^{\beta}}\right)^{1-2 \alpha}=K_{4} \frac{2^{-2 r H}}{n^{2 \beta H}} .
\end{aligned}
$$

Lemma 3.2 For $s, t \in\left[0,2^{-r}\right]$ such that $s<t$ and $\frac{n}{4} \leq r \leq \frac{n}{2}$, let $\rho(s, t)$ and $\rho^{\prime}(s, t)$ be, respectively, the correlations of $\left(X_{1}(s), X_{1}(t)\right)$ and $(X(s), X(a, b, t))$ (and, therefore, of $\left.\left(X_{1}(t), X_{1}(a, b, s)\right)\right)$. We have, for $\gamma$, the constant of Lemma 3.1,

(i)

$$
\left|\rho(s, t)-\rho^{\prime}(s, t)\right| \leq K_{3,2} \frac{2^{-2 r H}}{n^{\beta \gamma}} \frac{1}{s^{H} t^{H}}
$$

for $K_{3,2}<\infty$

(ii)

$$
1-\rho^{2}(s, t) \geq c_{1}\left|\frac{t-s}{t}\right|^{2 H}
$$

Proof Remark that $\sigma\left(X_{1}(t)\right)=\sigma\left(X_{1}(a, b, t)\right)$. The first property comes from Lemma 3.1 and the fact that

$$
\begin{aligned}
\rho(s, t)-\rho^{\prime}(s, t) & =\frac{E\left[X_{1}(s) X_{1}(t)\right]-E\left[X_{1}(s) X_{1}(a, b, t)\right]}{\sigma\left(X_{1}(s)\right) \sigma\left(X_{1}(t)\right)} \\
& =\frac{E\left[\hat{X}_{1}(a, b, s) \hat{X}_{1}(a, b, t)\right]}{s^{H} t^{H}} .
\end{aligned}
$$

If $\Sigma$ denotes the covariance matrix of $\left(X_{1}(s), X_{1}(t)\right)$, then

$$
\begin{aligned}
\operatorname{det}(\Sigma) & =\operatorname{Var}\left(X_{1}(s)\right) \operatorname{Var}\left(X_{1}(t)\right)\left(1-\rho^{2}(s, t)\right) \\
& =\operatorname{Var}\left(X_{1}(s)\right) \operatorname{Var}\left(X_{1}(t) \mid X_{1}(s)\right),
\end{aligned}
$$


and the second statement of the lemma is immediate given the strong LND property.

Theorem 3.3 Let $L\left[0,2^{-r}\right]$ and $L_{a, b}\left[0,2^{-r}\right]$ be, respectively, the local times of $X(\cdot)$ and $X(a, b, \cdot)$ at zero on $\left[0,2^{-r}\right]$. If $b=n^{\beta} 2^{-r}, a=2^{-r} / n^{\beta}$, and $\gamma=2 \min (H, 1-$ $H)$, then there exists a constant $K_{3,3}>0$ such that

$$
E\left[\left(L\left[0,2^{-r}\right]-L_{a, b}\left[0,2^{-r}\right]\right)^{2}\right] \leq K_{3,3} \frac{2^{-2 r(1-d H)}}{n^{\beta \gamma / 12}} .
$$

Proof First, by scaling, it is enough to show the result for $r=0$.

Let $P_{s, t}$ be the joint density of $\left(X_{1}(s), X_{1}(t)\right)$, and $P_{s, t}^{\prime}$ that of $\left(X_{1}(s), X_{1}(a, b, t)\right)$. Both pairs are Gaussian centered vectors, so their densities at $(0,0)$ are simply the reciprocal of the square roots of the determinants of the covariance matrices times $\frac{1}{2 \pi}$,

$$
\begin{aligned}
& P_{s, t}(0,0)=\left(\frac{1}{2 \pi} \frac{1}{\sigma\left(X_{1}(s)\right) \sigma\left(X_{1}(t)\right)} \frac{1}{\sqrt{1-\rho^{2}}}\right)^{d}, \quad \rho=\rho\left(X_{1}(s), X_{1}(t)\right), \\
& P_{s, t}^{\prime}(0,0)=\left(\frac{1}{2 \pi} \frac{1}{\sigma\left(X_{1}(s)\right) \sigma\left(X_{1}(a, b, t)\right)} \frac{1}{\sqrt{1-\rho^{\prime 2}}}\right)^{d}, \quad \rho^{\prime}=\rho\left(X_{1}(s), X_{1}(a, b, t)\right),
\end{aligned}
$$

and

$$
\begin{aligned}
E\left[\left(L[0,1]-L_{a, b}[0,1]\right)^{2}\right] & =2 \int_{0}^{1} \int_{0}^{1}\left[P_{s, t}(0,0)-P_{s, t}^{\prime}(0,0)\right] d t d s \\
& =4 \int_{0}^{1} \int_{s}^{1}\left[P_{s, t}(0,0)-P_{s, t}^{\prime}(0,0)\right] d t d s .
\end{aligned}
$$

We note that $P_{s, t}(0,0) \geq P_{s, t}^{\prime}(0,0)$.

We use two bounds on the integrand. For small values of $s$ and $|s-t|$, we will simply use $P_{s, t}(0,0)-P_{s, t}^{\prime}(0,0) \leq P_{s, t}(0,0)$; for other $(s, t)$, we use

$$
\begin{aligned}
P_{s, t}(0,0)-P_{s, t}^{\prime}(0,0) & \leq\left(\frac{1}{2 \pi}\right)^{d} \frac{1}{s^{d H}} \frac{1}{t^{d H}}\left(\left(\frac{1}{\sqrt{1-\rho^{2}}}\right)^{d}-\left(\frac{1}{\sqrt{1-\left(\rho^{\prime}\right)^{2}}}\right)^{d}\right) \\
& \leq\left(\frac{1}{2 \pi}\right)^{d} \frac{d}{s^{d H}} \frac{1}{t^{d H}}\left(\frac{1}{\sqrt{1-\rho^{2}}}\right)^{d} \frac{\rho\left|\rho-\rho^{\prime}\right|}{\left(1-\rho^{2}\right)^{3 / 2}} \\
& =P_{s, t}(0,0) \frac{d \rho\left|\rho-\rho^{\prime}\right|}{\left(1-\rho^{2}\right)^{3 / 2}}
\end{aligned}
$$

The key point is the easily verified consequence of Lemma 3.2 that outside $A=$ $\left\{(s, t): s<t,|s|^{H}<n^{-\beta \gamma / 3}\right.$ or $\left.\left(\frac{|t-s|}{t}\right)^{2 H}<n^{-\beta \gamma / 6}\right\}, \frac{\rho\left|\rho-\rho^{\prime}\right|}{\sqrt{\left.1-\rho^{2}\right)}}<\frac{K_{3,2}}{n^{\beta \gamma / 12}}$ for large $n$. Thus,

$$
E\left[\left(L[0,1]-L_{a, b}[0,1]\right)^{2}\right] \leq 4 \int_{A} P_{s, t}(0,0) d t d s+4 \int_{A^{c}} \frac{K_{2,3} P_{s, t}(0,0)}{n^{\beta \gamma / 12}} d t d s,
$$

from which the claimed inequality follows immediately. 
The next proposition expresses how well $L_{a, b}\left[0,2^{-r}\right]$ approximates $L\left[0,2^{-r}\right]$.

Proposition 3.4 With the same parameters as before, and $L\left[0,2^{-r}\right]$ and $L_{a, b}\left[0,2^{-r}\right]$ defined as above, we have for $n / 4 \leq r \leq n / 2$ large enough,

$$
P\left\{\left|L\left[0,2^{-r}\right]-L_{a, b}\left[0,2^{-r}\right]\right|>2^{-r(1-d H)}\right\} \leq \frac{K_{3,4}}{n^{\beta \gamma / 12}}
$$

for finite $K_{3,4}$.

The result follows from Chebyshev's inequality.

With the last results, we will prove the following:

Proposition 3.5 For all $\epsilon>0$ with $V=\left\{n_{i}, i>1\right\}$, the corresponding sequence of integers as in Proposition 2.5, and for $i>1$ sufficiently large,

$$
\begin{aligned}
& P\left\{\nexists \frac{n_{i}}{4} \leq r \leq \frac{n_{i}}{2}: 2^{-r(1-d H)}\left(\left(\log n_{i}\right)^{d H}+1\right) \leq\left(c_{0}+\epsilon\right)^{d H} L\left(0,2^{-r}\right)\right\} \\
& \quad \leq K_{3,5} \frac{n_{i}^{1-\beta \gamma / 12}}{\log n_{i}}
\end{aligned}
$$

for finite $K_{3,5}$.

Proof Let $\epsilon>0$ and $n_{i} \in V$, where $V$ is the sequence in Proposition 2.5. For $k_{0} \geq 0$ large enough, we define the sequence $r_{k}, 0 \leq k \leq k_{0}$, by

$$
\begin{aligned}
r_{0} & =n_{i} / 4, \\
r_{k+1} & =r_{k}+4 \beta \log n_{i} \quad \text { for } k \geq 1,
\end{aligned}
$$

until $r_{k_{0}}$ becomes more than $n_{i} / 2$. Then

$$
k_{0} \geq \frac{n_{i}}{16 \beta \log n_{i}}-1
$$

Thus, for $k \leq k_{0}$, we have $r_{k} \leq n_{i} / 2$. It is enough to prove that, for some $K$,

$$
P\left\{\nexists k<k_{0}:\left(\left(\log n_{i}\right)+1\right)^{d H} 2^{-r_{k}(1-d H)} \leq\left(c_{0}+\epsilon\right)^{d H} L\left(0,2^{-r_{k}}\right)\right\} \leq K \frac{n_{i}^{1-\beta \gamma / 12}}{\log n_{i}} .
$$

In order to create independence, let us consider the sequence $a_{k}=\frac{2^{-r_{k}}}{n_{i}^{\beta}}, b_{k}=$ $2^{-r_{k}} n_{i}^{\beta}$, and the processes $X_{k}(t)=X\left(a_{k}, b_{k}, t\right)$. As $k$ varies, these processes are independent. Moreover, we have from the scaling of the process and Proposition 2.5 that for all $k \geq 0$, there exists $\delta>0$ such that

$$
P\left\{\left(\frac{\log n_{i}}{c_{0}+\epsilon / 2}\right)^{d H} 2^{-r_{k}(1-d H)} \leq L_{k}\left(0,2^{-r_{k}}\right)\right\} \geq \frac{1}{n_{i}^{1-\delta}}
$$


where $L_{k}$ is the local time of the process $X_{k}$. By independence,

$$
\begin{aligned}
P\left\{\nexists k \leq k_{0}:\left(\frac{\log n_{i}}{c_{0}+\epsilon / 2}\right)^{d H} 2^{-r_{k}(1-d H)} \leq L_{k}\left(0,2^{-r_{k}}\right)\right\} & \leq\left(1-\frac{1}{n_{i}^{1-\delta}}\right)^{k_{0}} \\
& \leq \exp \left(-\frac{k_{0}}{n_{i}^{1-\delta}}\right) .
\end{aligned}
$$

Proposition 3.4 now implies

$$
P\left\{\left|L\left(0,2^{-r_{k}}\right)-L_{k}\left(0,2^{-r_{k}}\right)\right| \geq 2^{-r_{k}(1-d H)}\right\} \leq \frac{K_{3,4}}{n_{i}^{\beta \gamma / 12}} .
$$

Combining the last two results, we have

$$
\begin{aligned}
& P\left\{\nexists k \leq k_{0}:\left(\left(\log n_{i}\right)^{d H}\right) 2^{-r_{k}(1-d H)} \leq\left(c_{0}+\epsilon\right)^{d H} L\left(0,2^{-r_{k}}\right)\right\} \\
& =P\left\{\nexists k \leq k_{0}:\left(\left(\log n_{i}\right)^{d H}\right) 2^{-r_{k}(1-d H)} \leq\left(c_{0}+\epsilon\right)^{d H} L\left(0,2^{-r_{k}}\right) ;\right. \\
& \quad \forall k<k_{0}: \mid L\left(0,2^{-r_{k}}\right)-L_{k}\left(0,\left[0,2^{-r_{k}}[) \mid<2^{-r_{k}(1-d H)}\right\}\right. \\
& \quad+P\left\{\nexists k<k_{0}:\left(\left(\log n_{i}\right)^{d H}\right)\right) 2^{-r_{k}(1-d H)} \leq\left(c_{0}+\epsilon\right)^{d H} L\left(0,2^{-r_{k}}\right) ; \\
& \quad \forall k<k_{0}: \mid L\left(0,2^{-r_{k}}\right)-L_{k}\left(0,\left[0,2^{-r_{k}}[) \mid>2^{-r_{k}(1-d H)}\right\}\right. \\
& \leq P\left\{\nexists k<k_{0}:\left(\left(\log n_{i}\right)^{d H}-2\right) 2^{-r_{k}(1-d H)} \leq\left(c_{0}+\epsilon\right)^{d H} L_{k}\left(0,2^{-r_{k}}\right)\right\}+k_{0} \frac{K_{3,4}}{n_{i}^{\beta \gamma / 12}} \\
& \leq \exp \left(-\frac{k_{0}}{n_{i}^{1-\delta}}\right)+k_{0} \frac{K_{3,4}}{n_{i}^{\beta \gamma / 12}} \\
& \leq K_{3,1} \frac{n_{i}^{1-\beta \gamma / 12}}{\log n_{i}} .
\end{aligned}
$$

\section{Upper Bound for the Hausdorff Measure}

As before, $\{X(t), t \in \mathbb{R}\}$ is a $d$-dimensional fractional Brownian motion of index $H$ $(0<H<1)$. In this section we obtain the upper bound for the Hausdorff $\phi$-measure. We show that for $c_{0}$, the constant in Proposition 2.5,

$$
m_{\phi}\left(X^{-1}(0) \cap[0,1]\right) \leq c_{0}^{d H} L(0,1) \quad \text { with } \phi(r)=r^{1-d H}\left(\log \log \frac{1}{r}\right)^{d H} .
$$

In order to do this, it is sufficient to "produce" a sequence of asymptotically efficient coverings.

Fix $\epsilon>0, z>0$, and let $Z=[z, 1]$. Using the continuity of local time, it is sufficient to prove that

$$
m_{\phi}\left(X^{-1}(0) \cap Z\right) \leq\left(c_{0}+\epsilon\right)^{d H} L(0, Z) .
$$


We will use an approach similar to that of [28] and [21]. They consider intervals of different sizes to construct an optimal covering of the zero set, $A=\{t \in Z: X(t)=$ $0\}$. The covering intervals are classified as either good or bad.

For a fixed $n$, consider the collection $I_{n}^{z}$ of intervals $I_{i, n}^{z}=\left[i / 2^{n},(i+1) / 2^{n}\right) \cap Z$, $i=\left[z 2^{n}\right],\left[z 2^{n}\right]+1, \ldots I_{i, n}^{z}$ is a good covering interval if

$$
\begin{aligned}
& 0 \in X\left(I_{i, n}^{z}\right) \quad \text { and } \quad \exists \frac{n}{4} \leq r \leq \frac{n}{2} \quad \text { such that } \\
& \quad \phi\left(2^{-r}\right) \leq\left(c_{0}+\epsilon\right)^{d H} L\left(0,\left[i / 2^{n}, i / 2^{n}+2^{-r}\right]\right) .
\end{aligned}
$$

For a good interval, we replace $I_{i, n}^{z}$ by $I_{i, n}^{z, r_{i}}=\left[i / 2^{n}, i / 2^{n}+2^{-r_{i}}\right)$, where $r_{i}$ is the largest $r$ satisfying $\phi\left(2^{-r}\right) \leq\left(c_{0}+\epsilon\right)^{d H} L\left(0, I_{i, n}^{z, r}\right)$. The next interval in the covering is the first good interval beginning at $i / 2^{n}+2^{-r_{i}}$.

In order to complete the covering, we must include some bad intervals. We say that $I_{i, n}^{z}$ is bad if

$$
0 \in X\left(I_{i, n}^{z}\right) \quad \text { and } \quad \nexists \frac{n}{4} \leq r \leq \frac{n}{2} \quad \text { such that } \quad \phi\left(2^{-r}\right) \leq\left(c_{0}+\epsilon\right)^{d H} L\left(0, I_{i, n}^{z, r}\right) .
$$

The main problem is to show that the contribution to the covering from bad intervals is small.

Let $T_{n}^{z}$ denote the number of bad intervals $I_{i, n}^{z}$ with $\left[z 2^{n}\right] \leq i \leq 2^{n}$. The proof of the upper bound is essentially reduced to the proof of the following theorem.

Theorem 4.1 With probability one, there exists an increasing sequence of integers $\left\{n_{j} ; j>1\right\}$ going to infinity and $j_{0}>0$ such that

$$
\forall j>j_{0}: \phi\left(2^{-n_{j}}\right) \times T_{n_{j}}^{z}<1 / n_{j}
$$

Remark This means that the covering of bad intervals must make contribution $=$ $T_{n_{i}}^{z} \phi\left(2^{-n_{i}}\right)$, which is negligible.

Proof According to Proposition 3.5, given $\epsilon>0$, there exists an increasing sequence $V=\left\{n_{i} ; i>1\right\}$ of positive integers with infinite limit such that, for all large $n_{i} \in V$, we have

$$
\begin{aligned}
& P\left\{\nexists \frac{n}{4} \leq r \leq \frac{n}{2}: 2^{-r(1-d H)}\left((\log n)^{d H}+1\right) \leq\left(c_{0}+\epsilon\right)^{d H} L\left(0,2^{-r}\right)\right\} \\
& \quad \leq K_{3,2} \frac{n^{1-\beta \gamma / 12}}{\log n} .
\end{aligned}
$$

Fix $n \in V$. Note now that event $\left\{I_{i, n}^{z}\right.$ is bad $\}$ is contained in

$$
\left\{\exists t \in I_{i, n}^{z} \cap A ; \nexists \frac{n}{4} \leq r \leq \frac{n}{2}: \phi\left(2^{-r}\right) \leq\left(c_{0}+\epsilon\right)^{d H} L\left(X(t), I_{i, n}^{z, r}\right)\right\} .
$$


We introduce the following events:

$$
\begin{aligned}
& R_{i, n}=\left\{\sup _{s, t \in I_{i, n}^{z}}|X(t)-X(s)|<n 2^{-n H}\right\} ; \\
& T_{i, n}=\left\{\sup _{s \in I_{i, n}^{z}}\left|L\left(X(s), I_{i, n}^{z, r}\right)-L\left(X\left(\frac{i}{2^{n}}\right), I_{i, n}^{z, r}\right)\right|<2^{-r(1-d H)}\right\} ; \\
& S_{i, n}=\left\{\nexists \frac{n}{4} \leq r \leq \frac{n}{2}: \phi\left(2^{-r}\right)+2^{-r(1-d H)} \leq\left(c_{0}+\epsilon\right)^{d H} L\left(X\left(\frac{i}{2^{n}}\right), I_{i, n}^{z, r}\right)\right\} .
\end{aligned}
$$

By Lemma 2.1 and Proposition 2.4,

$$
P\left\{R_{i, n}^{c}\right\} \leq \exp \left(-n^{2} / K_{2,2}\right) \quad \text { and } \quad P\left\{T_{i, n}^{c}\right\} \leq \exp \left(-n^{2} / K_{2,3}\right) \text {. }
$$

For $S_{i, n}$, using Proposition 3.5 and the fact that $\phi\left(2^{-r}\right) \leq 2^{-r(1-d H)}(\log n)^{d H}$, we have

$$
P\left\{S_{i, n}\right\} \leq K_{3,2} \frac{n^{1-\beta \gamma / 12}}{\log n}
$$

$$
\begin{aligned}
P\left\{I_{i, n}^{z} \text { is bad }\right\}= & P\left\{I_{i, n}^{z} \text { is bad; } R_{i, n} ; T_{i, n}\right\}+P\left\{I_{i, n}^{z} \text { is bad; }\left(R_{i, n} \cap T_{i, n}\right)^{c}\right\} \\
\leq & P\left\{\exists t \in I_{i, n}^{z} \cap A ; S_{i, n} ; R_{i, n} ; T_{i, n}\right\}+P\left\{\left(R_{i, n} \cap T_{i, n}\right)^{c}\right\} \\
\leq & P\left\{\exists t \in I_{i, n}^{z} \cap A \mid S_{i, n} ; R_{i, n} ; T_{i, n}\right\} P\left\{S_{i, n} ; R_{i, n} ; T_{i, n}\right\} \\
& +\exp \left(-n^{2} / K_{2,2}\right)+\exp \left(-n^{2} / K_{2,3}\right) .
\end{aligned}
$$

For the first probability in the right part of the last inequality, we use the strong LND property of the process $X$ since

$$
\begin{gathered}
S_{i, n}, T_{i, n}, \text { and } R_{i, n} \text { all are in } \sigma\left(X(t)-X\left(\frac{i}{2^{n}}\right) ; t \geq \frac{i}{2^{n}}\right) . \\
P\left\{\exists t \in I_{i, n}^{z} \cap A \mid S_{i, n} ; R_{i, n} ; T_{i, n}\right\}=P\left\{\left|X\left(\frac{i}{2^{n}}\right)\right| \leq n 2^{-n H} \mid S_{i, n} ; R_{i, n} ; T_{i, n}\right\} \\
\leq \frac{c_{1}^{d}}{z^{d H}}(2 n)^{d} 2^{-n d H}
\end{gathered}
$$

for the constant $c_{1}$ of (1.4). Then

$$
P_{i}=P\left\{I_{i, n}^{z} \text { is bad }\right\} \leq K \frac{2^{-n d H} n^{d+1-\beta \gamma / 12}}{z^{d H} \log n}
$$

for some universal $K$. We have 


$$
\begin{aligned}
E\left[\phi\left(2^{-n}\right) T_{n}^{z}\right] & =2^{-n(1-d H)}\left(\log \log 2^{n}\right)^{d H} E\left[T_{n}^{z}\right] \\
& =2^{-n(1-d H)}(\log (n \log 2))^{d H} \sum_{i} P\left\{I_{i, n}^{z} \text { is bad }\right\} \\
& \leq 2^{-n(1-d H)}(\log (n \log 2))^{d H} 2^{n} P_{1} \\
& \leq 2^{n H}(\log (n \log 2))^{d H} K \frac{2^{-n H}}{z^{d H} \log n} n^{d+1-\beta \gamma / 12} \\
& \leq \frac{K}{z^{d H}} \frac{(\log (n \log 2))^{d H}}{\log n} \frac{1}{n^{\beta \gamma / 12-d-1}}
\end{aligned}
$$

and

$$
P\left(\phi\left(2^{-n}\right) \times T_{n}^{z}>\frac{1}{n}\right) \leq K n^{d+2-\beta \gamma / 12} \frac{1}{z^{d H}} .
$$

With a choice of $\beta>36(d+2) \frac{2}{\min (1-H, H)}$ and the Borel-Cantelli lemma, we have proven that the contribution of bad intervals is small.

If $\sum_{I}$ and $\sum_{I I}$ are, respectively, the contributions of good and bad intervals, there exist an increasing sequence of integers $\left\{n_{j} ; j>1\right\}$ and $j_{0}>0$ such that, for all $j>j_{0}$, we have

$$
\begin{aligned}
\sum_{i} \phi\left(\left|I_{i, n_{j}}^{z}\right|\right) & =\sum_{I} \phi\left(\left|I_{i, n}^{z, r_{i}}\right|\right)+\sum_{I I} \phi\left(\left|I_{i, n}^{z}\right|\right) \\
& \leq\left(c_{0}+\epsilon\right)^{d H} L\left(0,\left[z, 1+2^{-n_{j} / 4}\right]\right)+\frac{1}{n_{j}} .
\end{aligned}
$$

By continuity,

$$
\text { for all } z, \epsilon>0, \quad m_{\phi}\left(X^{-1}(0) \cap[z, 1]\right) \leq\left(c_{0}+\epsilon\right)^{d H} L(0,[z, 1]) .
$$

By the continuity of local time and Hausdorff $\phi$-measure, the proof is complete.

Remark The argument given deals explicitly with bounds for the level set $X^{-1}(0)$, but for treating general $X^{-1}(x)$, not many changes are needed. The argument basically began by considering the intervals $\left[\frac{i}{2^{n}}, \frac{i+1}{2^{n}}\right)$ and classing the good or bad according to the behavior of local times of $\left(X\left(\frac{i}{2^{n}}+s\right)-X\left(\frac{i}{2^{n}}\right): s \geq 0\right)$. This in no way depended on the value of $X\left(\frac{i}{2^{n}}\right)$. Then a bound on the probability that "bad" intervals were relevant to $X^{-1}(0)$ was achieved via a use of the strong LND property, which applies equally well to $X^{-1}(x)$.

\section{Lower Bound for the Hausdorff Measure}

In order to show the lower bound for the Hausdorff measure, it suffices to prove that

$$
\forall \epsilon>0 \text { and } z>0: \frac{c_{0}^{d H}}{1+2 \epsilon} L(0, Z) \leq m_{\phi}\left(X^{-1}(0) \cap Z\right),
$$

where $c_{0}$ is the constant in Proposition 2.5, and, again, $Z=[z, 1]$. 
Fix such $\epsilon$ and $z$.

Proposition 5.1 There exists a constant $\delta>0$ such that, for all $j$ large enough,

$$
P\left\{L\left(0,(1+\epsilon)^{-j}\right) \geq c_{0}^{-H d}(1+\epsilon) \phi\left((1+\epsilon)^{-j}\right)\right\} \leq \frac{1}{j^{1+\delta}} .
$$

Proof Recall that $c_{0}$ is the constant defined in Proposition 2.5 by

$$
c_{0}=\liminf _{x \rightarrow \infty} \frac{-\log P\{L(0,1)>x\}}{x^{1 / d H}} .
$$

So, for $x$ large enough,

$$
\frac{-\log P\{L(0,1)>x\}}{x^{1 / d H}} \geq \frac{c_{0}}{1+\epsilon} \Rightarrow P\{L[0,1] \geq x\} \leq e^{-\frac{c_{0}}{(1+\epsilon)} x^{1 / d H}} .
$$

Thus, for $j$ large enough,

$$
\begin{aligned}
& P\left\{L\left(0,(1+\epsilon)^{-j}\right) \geq c_{0}^{-d H}(1+\epsilon) \phi\left((1+\epsilon)^{-j}\right)\right\} \\
& \quad \leq P\left\{L\left(0,(1+\epsilon)^{-j}\right) \geq c_{0}^{-d H}(1+\epsilon)(1+\epsilon)^{-j(1-d H)}\left(\log \log (1+\epsilon)^{j}\right)^{d H}\right\} \\
& \quad=P\left\{L(0,1) \geq c_{0}^{-d H}(1+\epsilon)(\log j)^{d H}\right\} \\
& \quad \leq e^{-\frac{c_{0}}{1+\epsilon} c_{0}^{-d H}(1+\epsilon)^{1 / d H} \log j}=\left(\frac{1}{j}\right)^{(1+\epsilon)^{(1-d H) / H}} .
\end{aligned}
$$

Let $\delta=(1+\epsilon)^{(1-d H) / H}$; then

$$
P\left\{L\left(0,(1+\epsilon)^{-j}\right) \geq c_{0}^{-d H}(1+\epsilon) \phi\left((1+\epsilon)^{-j}\right)\right\} \leq \frac{1}{j^{1+\delta}} .
$$

For $n, j>0$, the intervals $\left[\frac{i}{2^{n}}, \frac{i+1}{2^{n}}\right]$ and $\left[\frac{i}{2^{n}}, \frac{i}{2^{n}}+(1+\epsilon)^{-j}\right]$ will be denoted by $I_{i, n}$ and $I_{i, n}^{j}$. An interval $I_{i, n}$ will be called $j$-bad for the sample point $\omega$ if

(i) $\exists s \in I_{i, n}$ such that $X(s)=0$;

(ii) $\exists t \in I_{i, n}$ such that $L\left(X(t), I_{i, n}^{j}\right)>c_{0}^{-d H}(1+2 \epsilon) \phi\left((1+\epsilon)^{-j}\right)$.

Proposition 5.2 For all $j>0$ large enough, $n>0$ such that $(1+\epsilon)^{-j} \in$ $\left[2^{-n / 2}, 2^{-n / 4}\right]$, and for $i / 2^{n} \in Z$, there exists $\delta>0$ such that

$$
P\left(I_{i, n} \text { is } j-b a d\right) \leq \frac{K}{j^{1+\delta}},
$$

where $K>0$ is a constant independent of $i$ and $n$. 
Proof Let $j>0$. If $n$ and $i \in\left\{1,2, \ldots, 2^{n}\right\}$ are such that $(1+\epsilon)^{-j} \in\left[2^{-n / 2}, 2^{-n / 4}\right]$ and $i / 2^{n} \in Z$, then, using Proposition 2.4, we have that

$$
P\left\{\sup _{s \in I_{i, n}}\left|L\left(X(s), I_{i, n}^{j}\right)-L\left(X\left(i / 2^{n}\right), I_{i, n}^{j}\right)\right| \geq(1+\epsilon)^{-j(1-d H)}\right\} \leq \exp \left(-n^{2} / K_{2,3}\right) .
$$

So,

$$
\begin{aligned}
& P\left\{I_{i, n} \text { is } j \text {-bad }\right\} \\
& =P\left\{I_{i, n} \text { is } j\right. \text {-bad, and } \\
& \left.\quad \sup _{s \in I_{i, n}}\left|L\left(X(s), I_{i, n}^{j}\right)-L\left(X\left(i / 2^{n}\right), I_{i, n}^{j}\right)\right| \leq(1+\epsilon)^{-j(1-d H)}\right\} \\
& \quad+P\left\{I_{i, n} \text { is } j\right. \text {-bad, and } \\
& \left.\quad \sup _{s \in I_{i, n}}\left|L\left(X(s), I_{i, n}^{j}\right)-L\left(X\left(i / 2^{n}\right), I_{i, n}^{j}\right)\right| \geq(1+\epsilon)^{-j(1-d H)}\right\} \\
& \leq P\left\{L\left(X\left(i / 2^{n}\right), I_{i, n}^{j}\right) \geq c_{0}^{-d H}(1+2 \epsilon) \phi\left((1+2 \epsilon)^{-j}\right)-(1+\epsilon)^{-j(1-d H)}\right\} \\
& \quad+e^{-n^{2} / K_{2,3}} \\
& \leq P\left\{L\left(X\left(i / 2^{n}\right), I_{i, n}^{j}\right) \geq c_{0}^{-d H}(1+\epsilon) \phi\left((1+\epsilon)^{-j}\right)\right\}+e^{-n^{2} / K_{2,3}} \\
& <\frac{K_{2}}{j^{1+\delta}}+e^{-n^{2} / K_{2,3}} \leq \frac{K^{1+\delta}}{j^{1+\delta}}
\end{aligned}
$$

Proposition 5.3 For all $n>0$ and $i / 2^{n} \in Z$, there exists a constant $h=h(z)>0$ such that

$$
E\left[L\left(0, I_{i, n}\right) \mid X(t)-X\left(i / 2^{n}\right), t \geq i / 2^{n}\right] \leq h 2^{-n}
$$

Proof Consider the process $(Y(t): t \geq 0)=\left(X\left(t+i / 2^{n}\right)-X\left(i / 2^{n}\right): t \geq 0\right)$. Then

$$
\begin{aligned}
E\left[L\left(0, I_{i, n}\right) \mid X(t)-X\left(i / 2^{n}\right), t \geq i / 2^{n}\right] & =E\left[L^{Y}\left(-X\left(i / 2^{n}\right)\right),\left[0,2^{-n}\right) \mid Y(\cdot)\right] \\
& =\int L^{Y}\left(y,\left[0,2^{-n}\right]\right) \cdot P\left(X\left(i / 2^{n}\right)\right. \\
& =-d y \mid Y(\cdot)) \\
& \leq h \int L^{Y}\left(y,\left[0,2^{-n}\right]\right) d y \\
& =h \cdot 2^{-n} .
\end{aligned}
$$

This last inequality follows since, thanks to the LND property, the conditional density of $X\left(i / 2^{n}\right)$, given $X\left(i / 2^{n}+s\right)-X\left(i / 2^{n}\right), s \geq 0$, is bounded, and $\int L^{Y}\left(y,\left[0,2^{-n}\right]\right) d y$ $=\left|I_{i, n}\right|=2^{-n}$. 
Theorem 5.4 With probability one, for $L(0, \cdot)$-almost all $t \in Z$,

$$
\limsup _{r \rightarrow 0} \frac{L(0,[t, t+r])}{\phi(r)} \leq c_{0}^{-d H}(1+2 \epsilon)(1+\epsilon) .
$$

Proof Let

$$
A_{j}=\left\{t \in Z: L\left(0,\left[t, t+(1+\epsilon)^{-j}\right]\right) \geq c_{0}^{-d H}(1+2 \epsilon) \phi\left((1+\epsilon)^{-j}\right)\right\},
$$

and let $n=n(j)$ be such that $(1+\epsilon)^{-j} \in\left[2^{-n / 2}, 2^{-n / 4}\right]$. We have

$$
A_{j} \subseteq \bigcup_{i / 2^{n}>z}\left\{I_{i, n} \text { is } j \text {-bad }\right\}
$$

and

$$
\begin{aligned}
E\left[L\left(A_{j}\right)\right] & \leq \sum_{i / 2^{n}>z} E\left[L\left(0, I_{i, n}\right) \mathcal{I}_{I_{i, n}(\text { is } j \text {-bad })}\right] \\
& \leq 2^{n} h 2^{-n} \frac{K}{j^{1+\delta}} \leq \frac{K_{1}}{j^{1+\delta}} .
\end{aligned}
$$

This implies that

$$
E\left[\sum_{j=1}^{\infty} L\left(A_{j}\right)\right]<\infty .
$$

Therefore, with probability 1 , for $L(0, \cdot)$-almost all $t \in Z$,

$$
\limsup _{j \rightarrow \infty} \frac{L\left(0,\left[t, t+(1+\epsilon)^{-j}\right]\right)}{\phi\left((1+\epsilon)^{-j}\right)} \leq c_{0}^{-d H}(1+2 \epsilon) .
$$

It is clear that (5.1) follows immediately from (5.2).

To conclude we need the following result from [28], Lemma 4, and [31], Theorem 4.1.

Theorem 5.5 Let $L(0, \cdot)$ be the local time of $X(t)$ at 0 , which is a random measure supported on $X^{-1}(0)$. Then, with probability 1 ,

$$
m_{\phi}\left(X^{-1}(0) \cap Z\right) \geq \frac{c_{0}^{d H}}{(1+2 \epsilon)(1+\epsilon)} L(0, Z) .
$$

Proof As already mentioned, $L(0, \cdot)$ is a locally finite Borel measure in $\mathbb{R}$ supported on $X^{-1}(0)$.

Let

$$
D=\left\{t \in Z: \limsup _{r \rightarrow 0} \frac{L(0,[t, t+r[)}{\phi(r)}>c_{0}^{-d H}(1+2 \epsilon)\right\} .
$$


Then, $D$ is a Borel set, and by Theorem $5.4, L(0, D)=0$ almost surely. Using the upper density theorem of Rogers and Taylor (1969), we have almost surely

$$
m_{\phi}\left(X^{-1}(0) \cap Z\right) \geq m_{\phi}\left(X^{-1}(0) \cap(Z \backslash D)\right) \geq \frac{c_{0}^{d H}}{1+2 \epsilon} L(0, Z \backslash D)=\frac{c_{0}^{d H}}{1+2 \epsilon} L(0, Z) .
$$

This completes the proof of Theorem 5.5.

Letting $z$ and $\epsilon$ tend to 0 , the proofs of the lower bound and of Theorem 1.2 are complete.

Remark Again, the extension of the argument to a general level set $X^{-1}(x)$, is more notational than theoretical. The argument considered the intervals $\left[\frac{i}{2^{n}}, \frac{i+1}{2^{n}}\right)$ and classed the good or bad according to the local times behavior of $\left(X\left(\frac{i}{2^{n}}+s\right)-X\left(\frac{i}{2^{n}}\right)\right.$ : $s \geq 0)$. Once more, this in no way depended on the value of $X\left(\frac{i}{2^{n}}\right)$. Then a bound on the probability that "bad" intervals were relevant to $X^{-1}(0)$ was achieved via a use of the strong LND property, which applies equally well to $X^{-1}(x)$.

\section{Temporal Dimension Greater than One}

We now sketch how to extend this result to higher temporal dimensions. There are several definitions, and we choose, for simplicity, Besicovitch's spherical Hausdorff measure (see, e.g., [15]) explicitly, but the result holds for other common definitions.

To show the higher-dimensional analogue of Theorem 5.4, the main change is that must show that, for say $I=[0,1]^{N}$ and $L(I)$-almost all $x$,

$$
\lim _{r \downarrow 0} \sup _{y \in B(x, r)} \frac{L(B(y, r))}{r^{N-H d}\left(\log \log \left(\frac{1}{r}\right)\right)^{\frac{H d}{N}}} \leq\left(\frac{1}{c_{0}}\right)^{\frac{H d}{N}},
$$

where now $c_{0}$ is defined to be

$$
\liminf _{x \rightarrow \infty} \inf _{y \in B(0,1)} \frac{-\log (P(L(B(y, 1))>x))}{x^{N / H d}},
$$

instead of simply $c_{0}=\liminf _{x \rightarrow \infty}-\frac{\log P\{L(0,1)>x\}}{x^{1 / d H}}$ as in the proof of Theorem 5.4. This, however, is easily dealt, given that, for any $\epsilon>0$, there are a finite number of radius $r(1+\epsilon)$ balls in $N$-space, $B_{1}, \ldots, B_{R}$, such that any radius $r$ ball containing the origin is strictly contained in $B_{i}$ for some $i \in\{1, \ldots, R\}$. From this we have in the usual way that

$$
m_{\phi}\left(\left\{\underline{t}: t \in[0,1]^{N}: X(\underline{t})=\underline{0}\right\}\right) \geq c_{0}^{\frac{d H}{N}} L\left(0,[0,1]^{N}\right) .
$$

The proof of the upper bound is a little more involved. As before, it is enough to show that, for $\epsilon, C>0$ but otherwise arbitrary.

$$
m_{\phi}\left(\underline{t}: \underline{t} \in[C, 1]^{N}, X(\underline{t})=\underline{0}\right) \leq c_{0}^{\frac{d H}{N}}(1+100 \epsilon) L\left([C, 1]^{N}\right) .
$$


We fix a large integer $V$ such that $\left(1-\frac{1}{5^{N}}\right)^{V}<\frac{\epsilon}{100}$. As in the proof of Proposition 3.5, we can find a fixed $y \in B(0,1)$ and a sequence of $n_{i}$ tending to infinity such that

$$
\frac{-\log \left(P\left(L(B(y, 1))>\log \left(n_{i}\right)\right)\right)}{\log \left(n_{i}\right)^{N / H d}} \leq c_{0}+\epsilon .
$$

We divide up $[C, 1]^{N}$ into cubes of length $2^{-n}$ for $n$ in the above sequence $n_{i}$.

For each cube $\prod_{j=1}^{N}\left[\frac{i_{j}}{2^{n}}, \frac{i_{j}+1}{2^{n}}\right], B(\underline{i}, n, h)$ will denote the ball centered at $\left(\frac{i_{1}}{2^{n}}, \frac{i_{2}}{2^{n}}, \ldots, \frac{i_{N}}{2^{n}}\right)+y 2^{-h}$ of radius $2^{-h}$. For $h$ in the interval $\left[\frac{n}{4}, \frac{n}{2}\right]$, these balls will contain the cube for $n$ large enough. We say that a cube $\prod_{j=1}^{N}\left[\frac{i_{j}}{2^{n}}, \frac{i_{j}+1}{2^{n}}\right]$ is bad if

(a) $\exists \frac{n}{4} \leq k \leq \frac{n}{2}$ such that, for some ball $B(\underline{i}, n, k)$,

$$
L(B(\underline{i}, n, k)) \geq 2^{-k(N-d H)}(\log n)^{\frac{d H}{N}} c_{0}^{-\frac{d H}{N}}
$$

(b) $\exists 1 \leq k \leq V$ such that $\forall \frac{n}{4}+\frac{(k-1)}{4 V} n+2 \leq h \leq \frac{n}{4}+\frac{k n}{4 V}-2 V$,

$$
L(B(\underline{i}, n, h)) \leq 2^{-h(N-d H)}(\log n)^{\frac{d H}{N}}\left(\frac{1}{c_{0}}\right)^{\frac{d H}{N}}(1-50 \epsilon) .
$$

As in Proposition 3.5, we easily have that the probability that a cube is bad and contains a point in $\{\underline{t}: X(\underline{t})=0\}$ is bounded by $\frac{2^{-d H n}}{n}$ for large $n$.

We can now construct a good covering of the level set $\left\{\underline{t} \in[C, 1]^{N}: X(\underline{t})=0\right\}$. We can deal with time points in the level set in bad cubes just as with temporal dimension one (though ultimately we have to replace the cubes by appropriate spheres of the same order). The difference is that, in higher dimensions, fitting the balls on which there are large local times is a little more complicated than with one-dimensional intervals. The advantage of the new definition of "bad" is that, for a good interval, there are many balls of many different scales containing the good cube on which the local time is appropriately large. More concretely, condition (b) assures that, for good cubes and for $1 \leq k \leq R$, there is a ball of length $\frac{n}{4}+\frac{(k-1)}{4 V} n+2 \leq h \leq \frac{n}{4}+\frac{k n}{4 V}-2 V$. As $k$ varies, the ratios of the scales for these different classes must (by our choice of $V$ ) be less than $\epsilon$. As we will see, this permits a choice of coverage which is the inflation by a factor $1+2 \epsilon$ of a disjoint collection of sets.

As in Proposition 3.5, we can start with the "first" covering consisting of the intervals $\left[\frac{i_{j}}{2^{n}}, \frac{i_{j}+1}{2^{n}}\right]$ which are bad and level 1 balls $B(\underline{i}, n, K)$ with $\frac{n}{4}+2 \leq K \leq$ $\frac{n}{4}+\frac{n}{4 V}-2 V$ and $L(B(\underline{i}, n, K)) \geq 2^{-K(N-d H)}(\log n)^{\frac{d H}{N}}\left(\frac{1}{c_{0}}\right)^{\frac{d H}{N}}(1-50 \epsilon)$.

Using Vitali's lemma, we can extract a finite disjoint subcollection of these balls

$$
B_{1}^{1}, B_{2}^{1}, \ldots, B_{R_{1}}^{1}
$$

such that $L\left(\bigcup_{i \leq R_{1}} B_{i}^{1}\right) \geq \frac{1}{5^{N}} L\left([C, 1]^{N}\right)$.

We now move to the second covering. 
(1) We retain the disjoint $B_{i}^{1}, i=1, \ldots, R_{1}$, and the bad cubes $J_{i}$. We now inflate by factor $1+2 \epsilon$ the $B_{i}^{1}$ to obtain $B_{i}^{1, \prime}$.

(2) We consider all $\underline{i}$ that are not bad and such that $I_{i}$ is not contained in $\bigcup_{j} B_{j}^{1}$. As before we take the largest ball $B(\underline{i}, h, n)$ such that $L(B(\underline{i}, h, n))>(1-$ $100 \epsilon) 2^{-(N H d) k} n^{\frac{H d}{N}}$ for $\frac{n}{4}+\frac{(1)}{4 V} n+2 \leq h \leq \frac{n}{4}+\frac{2 n}{4 V}-2 V$.

For such indices $\underline{i}$, we write $i \sim 2$.

This gives the second covering.

To prepare the third covering, we choose from the $B(i, h, n)$ sets a Vitali subset of disjoint elements $B_{1}^{2}, B_{2}^{2}, \ldots, B_{R_{2}}^{2}$ such that $L\left(\bigcup_{1}^{R_{2}} B_{i}^{2}\right) \geq \frac{1}{5^{N}} L\left((C, 1)^{N} / \bigcup_{j=1}^{R_{1}} B_{j}^{1}\right)$. We remove any elements that touch $B_{l}^{1}$. The key point is that any ball $B_{j}^{2}$ that is not disjoint from any ball $B_{l}^{1}$ will be contained in $B_{l}^{1, \prime}$.

We continue in this way. It is to be noted that the $B_{i}^{k}$ are disjoint for $k=1,2, \ldots$, $1 \leq i \leq R_{k}$.

Finally we come to the last covering: bad cubes, $\left\{B_{i}^{1}\right\}_{i=1}^{R_{1}},\left\{B_{i}^{2}\right\}_{i=1}^{R_{2}}, \ldots$, $\left\{B_{i}^{R_{V-1}}\right\}_{i=1}^{R_{V-1}}$, and $B(\underline{i}, h, n)$ such that $L(B(\underline{i}, h, n)) \geq(1-100 \epsilon) 2^{-(N-H d) h} n^{\frac{H d}{N}}$, $\frac{n}{4}+\frac{(V-1)}{4 V} n+2 \leq h \leq \frac{n}{4}+\frac{V n}{4 V}-2 V$.

We then extract the Vitali subset $B_{i}^{V}, i=1, \ldots, R_{V}$. We then put (unlike the previous usage) $B_{i}^{V, \prime}$ to be the 3-factor inflation of $B_{i}^{V}$.

We consider for the covering $M_{j}$ (= bad cubes) unioned with $\bigcup B_{j}^{i, \prime}, i=1, \ldots, V$, $j=1, \ldots, R_{i}$.

First, as in Theorem 4.1,

$$
E\left(\sum_{\underline{i} \text { bad }} \phi\left(\sqrt{N} 2^{-n}\right)\right) \stackrel{n \rightarrow \infty}{\rightarrow} 0 .
$$

Secondly, the balls $B_{j}^{i}$ are disjoint, and so

$$
\begin{aligned}
\sum \phi\left(\left(B_{j}^{i, \prime}\right)\right) \leq & (1+2 \epsilon)^{N-d H} \sum_{i=1}^{V-1} \sum_{j=1}^{R_{i}} \phi\left(B_{j}^{i}\right) . \\
& +\left[3^{N-d H}-(1+2 \epsilon)^{N-d H}\right] \sum_{j=1}^{R_{V}} \phi\left(B_{j}^{V}\right) \\
\leq & \frac{(1+2 \epsilon)^{N-d H}}{1-50 \epsilon} c_{0}^{d H / N} \sum_{i=1}^{V-1} \sum_{j=1}^{R_{i}}\left(L\left(B_{j}^{i}\right)\right) \\
& +\left[3^{N-d H}-(1+2 \epsilon)^{N-d H}\right] c_{0}^{d H / N} L\left(\bigcup_{j=1}^{R_{V}} B_{j}^{n}\right)
\end{aligned}
$$




$$
\begin{aligned}
\leq & \frac{(1+2 \epsilon)^{N-d H}}{1-50 \epsilon} c_{0}^{d H / N} L\left([C, 1]^{N}\right) \\
& +\left(3^{N-d H}-(1+2 \epsilon)^{N-d H}\right)\left(1-\frac{1}{5^{N}}\right)^{V} c_{0}^{d H / N} L\left((C, 1)^{N}\right) \\
\leq & (1+K \epsilon) c_{0}^{d H / N} L\left([C, 1]^{N}\right)
\end{aligned}
$$

if $V$ is sufficiently large, and we are done.

\section{References}

1. Adler, R.J.: The uniform dimension of the level sets of a Brownian sheet. Ann. Probab. 6, 509-515 (1978)

2. Adler, R.J.: The Geometry of Random Fields. Wiley, New York (1981)

3. Baraka, D.: Path properties of fractional Brownian motion. Thesis, E.P.F.L.

4. Baraka, D., Mountford, T.: A law of iterated logarithm for fractional Brownian motions. In: Lecture Notes in Mathematics, vol. 1934, pp. 161-179. Springer, Berlin (2008)

5. Berman, S.M.: Local times and sample function properties of stationary Gaussian processes. Trans. Am. Math. Soc. 137, 277-299 (1969)

6. Berman, S.M.: Gaussian sample functions: uniform dimension and Hölder conditions nowhere. Nagoya Math. J. 46, 63-86 (1972)

7. Berman, S.M.: Local nondeterminism and local times of Gaussian processes. Indiana Univ. Math. J. 23, 69-94 (1973)

8. Berman, S.M.: Spectral conditions for local nondeterminism. Stoch. Process. Appl. 27(1), 73-84 (1987)

9. Cuzick, J.: Local nondeterminism and the zeros of Gaussian processes. Ann. Probab. 6, $72-84$ (1978)

10. Cuzick, J., Du Peez, J.P.: Joint continuity of Gaussian local times. Ann. Probab. 10, 810-817 (1982)

11. Davies, P.L.: Local Hölder conditions for the local times of certain stationary Gaussian processes. Ann. Probab. 4, 277-298 (1976)

12. Davies, P.L.: The exact Hausdorff measure of the zero set of certain stationary Gaussian processes. Ann. Probab. 5, 740-755 (1977)

13. Ehm, W.: Sample function properties of multiparameter stable processes. Z. Wahrscheinlichkeitstheorie Verw. Geb. 56, 195-228 (1981)

14. Embrechts, P., Maejima, M.: Selfsimilar Processes. Princeton University Press, Princeton (2002)

15. Falconer, K.J.: Fractal Geometry-Mathematical Foundations and Applications. Wiley, New York (1990)

16. Geman, D., Horowitz, J.: Occupation densities. Ann. Probab. 8, 1-67 (1980)

17. Kahane, J.P.: Some Random Series of Functions, 2nd edn. Cambridge University Press, Cambridge (1985)

18. Kasahara, Y., Kono, N., Ogawa, T.: On tail probabilities of local times of Gaussian processes. Stoch. Process. Appl. 82, 15-21 (1999)

19. Monrad, D., Pitt, L.D.: Local nondeterminism and Hausdorff dimension. In: Progress in Probability and Statistics (Seminar on Stochastic Processes, vol. 13, p. 163-189. Birkhäuser, Boston (1987)

20. Mountford, T., Shieh, N., Xiao, Y.: Tail behaviour of local times for fractional Brownian motion (2008, in preparation)

21. Perkins, E.: The exact Hausdorff measure of the level sets of Brownian motion. Z. Wahrscheinlichkeitstheorie Verw. Geb. 58, 373-388 (1981)

22. Pitt, L.D.: Local times for Gaussian vector fields. Indiana Univ. Math. J. 27, 309-330 (1978)

23. Revuz, D., Yor, M.: Continuous Martingales and Brownian Motion, 3rd edn. Springer, New York (1999)

24. Rogers, C.A.: Hausdorff Measures. Cambridge University Press, Cambridge (1998)

25. Rosen, J.: Self-intersections of random fields. Ann. Probab. 12, 108-119 (1984)

26. Samorodnitsky, G., Taqqu, M.S.: Stable Non-Gaussian Random Processes: Stochastic Models with Infinite Variance. Stochastic Modeling. Chapman \& Hall, New York (1994) 
27. Talagrand, M.: Hausdorff measure of trajectories of multiparameter fractional Brownian motion. Ann. Probab. 23, 767-775 (1995)

28. Taylor, S.J., Wendel, J.G.: The exact Hausdorff measure of the zero set of a stable process. Z. Wahrscheinlichkeitstheorie Verw. Geb. 6, 170-180 (1966)

29. Xiao, Y.: Dimensions results for Gaussian vector fields and index- $\alpha$ stable fields. Ann. Probab. 23, 273-291 (1995)

30. Xiao, Y.: Hausdorff measure of the sample paths of Gaussian random fields. Osaka J. Math. 33, 895913 (1996)

31. Xiao, Y.: Hölder conditions for the local times and the Hausdorff measure of the level sets of Gaussian random fields. Probab. Theory Relat. Fields 109, 129-157 (1997)

32. Xiao, Y.: The packing measure of the trajectories of multiparameter fractional Brownian motion. Math. Proc. Camb. Philos. Soc. 135, 349-375 (2003)

33. Xiao, Y.: Strong local nondeterminism of Gaussian random fields and its applications. In: Lai, T.-L., Shao, Q.-M., Qian, L. (eds.) Asymptotic Theory in Probability and Statistics with Applications, pp. 136-176. Higher Education Press, Beijing (2007) 